\section{Influenza follicles and their buds as early diagnostic markers of influenza: typical images}

An article by Dr Kenzaka' in the 'Images in medicine' section of the Postgraduate Medical Journal reported that influenza follicles are noted in ATLAS SAKUMA. ${ }^{2}$ However, there is no reference to influenza follicles in Chapter VII: Influenza of ATLAS SAKUMA. It instead refers to lymph follicles, and their typical cases are presented in Chapter I: Adenovirus. Furthermore, in Case 11 of Chapter VII: Influenza, lymph follicles that were suspected to be due to adenovirus infection were presented. Miyamoto and Watanabe ${ }^{3}$ were the first in the world to report posterior pharyngeal wall follicles in influenza and named them influenza follicles. The statistical data and the morphological classification of influenza follicles reported by Kenzaka were all drawn from Miyamoto and Watanabe. ${ }^{3}$ Moreover, the mean duration between the onset and evaluation of novel influenza infections, as reported by Miyamoto and Watanabe, ${ }^{3}$ was erroneously cited, as underlined in the following sentence: 'The sensitivity and specificity of influenza follicles observed $7.8 \pm 5.3 \mathrm{~h}$ (range, 3-20 h; median, $5 \mathrm{~h}$ ) after onset were $95.4 \%$ and $98.4 \%$, respectively, for the seasonal influenza diagnosis'.

Kenzaka claims that ATLAS SAKUMA ${ }^{2}$ is available only in Japanese, with no English translation. However, Sakuma published an English translation ${ }^{4}$ in 2008, where pharyngeal observations in influenza infections were noted as follows: 'the manifestations of oral cavity, fauces and pharynx are not severe, and usually only slight redness and slight swelling are observed'. It is truly regrettable that Sakuma's contribution through the English-translated work is not duly acknowledged. Also, two corrections are needed in the citation listed as Reference 2 by Kenzaka: (i) the referenced ATLAS SAKUMA in Japanese should be the second edition and (ii) the name of the publisher should be Maruzen Planet. ${ }^{2}$ Kenzaka presents an image of a patient's

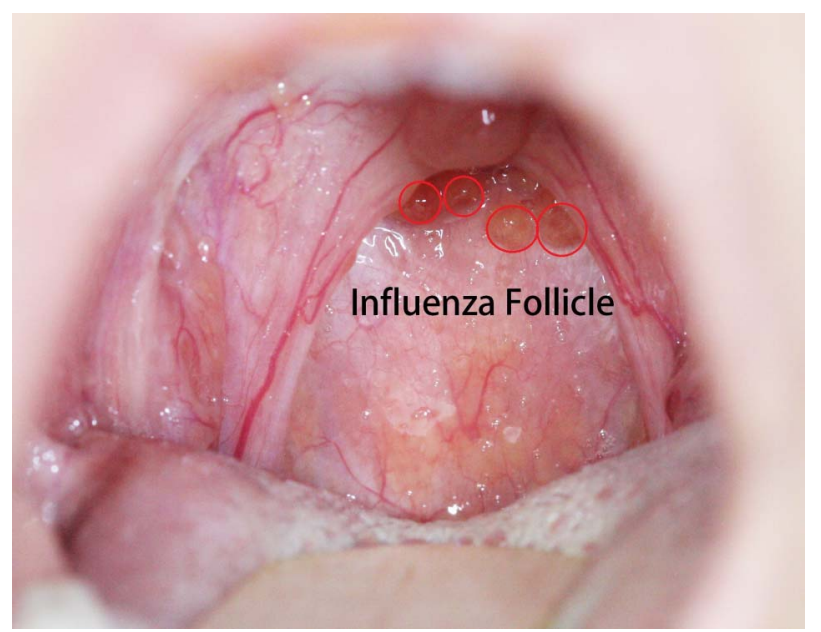

Figure 1 A typical image of a definitive influenza follicle according to Miyamoto and Watanabe' $s^{2}$ morphological classification of influenza follicles. In this case, the influenza follicles are aggregated in the upper part of the posterior pharyngeal wall; however, the location of the aggregation has no effect on the diagnosis.

pharynx with influenza follicles and describes the morphology of the influenza follicles to be Yamada/Fukutomi classification type II gastric polypoid lesions, as described by Miyamoto and Watanabe ${ }^{3}$ in General Medicine. According to the morphological classification of influenza follicles by Miyamoto and Watanabe, the lymph follicles of the posterior pharyngeal wall presented by Kenzaka are Yamada/Fukutomi classification type I gastric polypoid lesions; as such, they do not correspond to the definitive influenza follicles characterised by Miyamoto and Watanabe. These erroneous references and mischaracterisation of influenza follicles need to be corrected to avoid misunderstandings.

Among 419 patients diagnosed with seasonal influenza between 2003 and 2009, influenza follicles were identified with sensitivity of $94.5 \%$ and specificity of $98.4 \%$ regardless of the types of influenza $(\mathrm{A} / \mathrm{H} 3 \mathrm{~N} 2, \mathrm{~A} / \mathrm{H} 1 \mathrm{~N} 3$, and $\mathrm{B}){ }^{3}$ and the morphology of influenza follicles did not vary by the strains. Lymph follicles observed in upper respiratory inflammations such as adenovirus or enterovirus infection $^{2}$ are polymorphic and larger in size rather than hemispheric small (2$4 \mathrm{~mm}$ in diameter) follicles appearing in influenza infection, and influenza follicles are same in size for each patient. So, different sizes of influenza follicles observed in the same patient at the same time is a rare occurrence. Influenza follicles will be observed with or without associated severe lower airway disease.

A typical case of influenza follicles is shown in figure 1. Miyamoto and Watanabe also discovered the presence of smaller influenza follicles that can be seen during an earlier phase than the definitive follicles of influenza infection, and they named them influenza follicle buds ${ }^{5}$ (figure 2). This discovery may allow the diagnosis of influenza as early as 1 hour after fever onset, thereby enabling very early diagnosis of influenza and the identification of the pathophysiological expression of influenza follicles. 

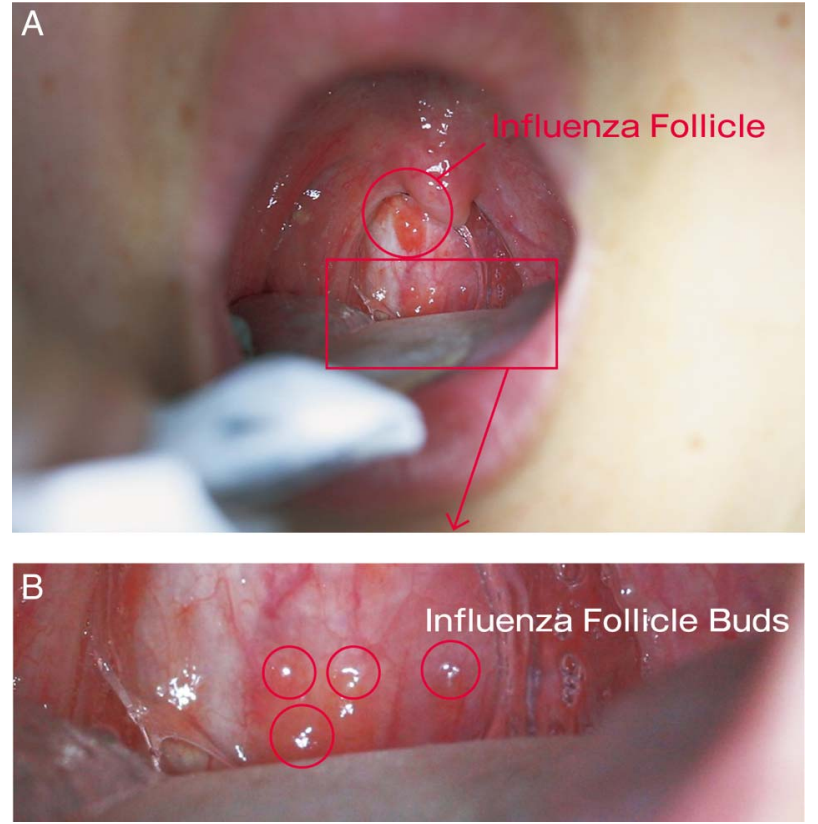

Figure 2 (A) A very rare case where influenza follicles and influenza follicle buds are observed together. Influenza follicle buds are shown inside the red rectangle. (B) A magnification of the image.

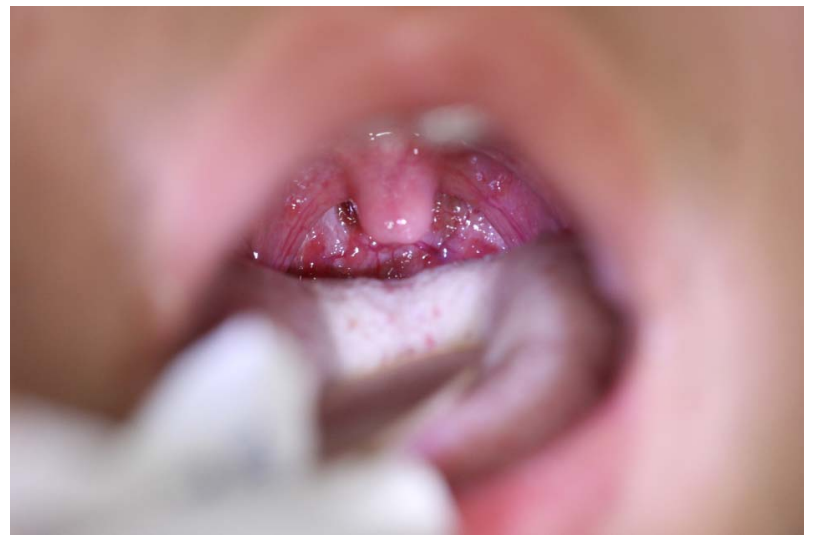

Figure 3 A typical image of influenza follicle buds. ${ }^{4}$

\section{Akihiko Miyamoto, ${ }^{1}$ Shigeyuki Watanabe ${ }^{2}$}

${ }^{1}$ Division of Clinical Medicine, Shoutokukai Medical Corporation, Sakuragawa-shi, Ibaraki-ken, Japan

${ }^{2}$ Tsukuba University Hospital Mito Medical Center, Mito Kyodo General Hospital, Graduate School of
Comprehensive Human Science, University of Tsukuba, Mito-shi, Japan

Correspondence to Dr Akihiko Miyamoto, Division of Clinical Medicine, Shoutokukai Medical Corporation,
Furushiro 229-1, Sakuragawa-shi, Ibaraki-ken 3004407, Japan; miyamo07@mocha.ocn.ne.jp

Competing interests None declared.

Patient consent Obtained.

Ethics approval Institutional review board at Tsukuba University Hospital, Mito Medical Center.

Provenance and peer review Not commissioned; internally peer reviewed.

- Additional material is published online only. To view please visit the journal online (http://dx.doi.org/10. 1136/postgradmedj-2016-134271).

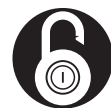

\section{OPEN ACCESS}

Open Access This is an Open Access article distributed in accordance with the Creative Commons Attribution Non Commercial (CC BY-NC 4.0) license, which permits others to distribute, remix, adapt, build upon this work non-commercially, and license their derivative works on different terms, provided the original work is properly cited and the use is noncommercial. See: http://creativecommons.org/licenses/ by-nc/4.0/

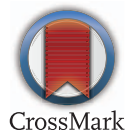

To cite Miyamoto A, Watanabe S. Postgrad Med J 2016;92:560-561.

Received 10 June 2016

Revised 1 July 2016

Accepted 4 July 2016

Published Online First 27 July 2016

Postgrad Med J 2016;92:560-561.

doi:10.1136/postgradmedj-2016-134271

\section{REFERENCES}

1 Kenzaka T. Influenza follicles in the posterior pharyngeal wall. Postgrad Med J 2015;91:472.

2 Sakuma T. ATLAS SAKUMA. 2nd edn. Tokyo, Japan: Maruzen Planet, 2008.

3 Miyamoto A, Watanabe S. Posterior pharyngeal wall follicles as early diagnostic marker for seasonal and novel influenza. Gen Med 2011;12:51-60.

4 Sakuma T. Infant and children's pharynx and skin with infectious diseases. Fukuoka, Japan: Shoshi Kankanbou, System Create Co., Ltd., 2008:87.

5 Miyamoto A, Watanabe S. Posterior pharyngeal wall follicles as a diagnostic marker of influenza during physical examination: considering their meaning and value. J Nihon Univ Med Assoc 2013;72:11-18. 